\title{
EFECTO DEL LEVONORGESTREL COMO ANTICONCEPTIVO ORAL DE EMERGENCIA EN LA OVULACIÓN, EL ENDOMETRIO Y LOS ESPERMATOZOIDES
}

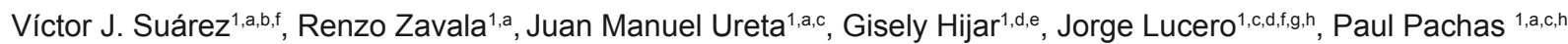

\begin{abstract}
RESUMEN
Existe amplia controversia acerca del mecanismo de acción del levonorgestrel como anticonceptivo oral de emergencia; numerosas organizaciones, tanto científicas como de la sociedad civil, muestran su disconformidad con su uso, debido a su posible acción como inductor de aborto. Con el objetivo de evaluar la evidencia científica disponible sobre los mecanismos de acción del levonorgestrel utilizado como anticonceptivo oral de emergencia (AOE), se realizó una revisión sistemática en las bases de datos Medline y Cochrane Library donde se encontró 444 artículos; después de revisar los resúmenes, se seleccionó 22 artículos, los cuales fueron evaluados a texto completo. Se encontró que el principal mecanismo de acción del levonorgestrel, a las dosis recomendadas como AOE, es la inhibición o retraso de la ovulación; no afecta a los espermatozoides en su capacidad de migración ni de penetración al óvulo. No se ha demostrado alteraciones morfológicas ni moleculares en el endometrio que puedan interferir con la implantación del huevo fecundado. No existe evidencia científica actual disponible que sustente que el uso de levonorgestrel como AOE sea abortivo.
\end{abstract}

Palabras clave: Anticoncepción postcoital; Levonorgestrel; Endometrio; Ovulación; Espermatozoides (fuente: DeCS BIREME).

\section{EFFECT OF LEVONORGESTREL IN THE OVULATION, ENDOMETRIUM, AND SPERMATOZOA FOR EMERGENCY ORAL CONTRACEPTION}

\begin{abstract}
There is wide controversy about the mechanism of action of the levonorgestrel used for emergency oral contraception, and many organizations, both scientific as well as from the civil society, show their discrepancy with its use, due to its possible action as an abortion- inducer. In order to evaluate the scientific evidence available on the mechanisms of action of the levonorgestrel used for emergency oral contraception (EOC), a systematic revision was performed in the Medline and Cochrane library databases. We found 444 articles. After reviewing the abstracts, we selected 22 articles, whose complete texts were evaluated. We found that the main mechanism of action of the levonorgestrel, given at the doses recommended for EOC, is the inhibition or retardation of the ovulation, it doesn't affect the spermatozoa in their migration or egg-penetration capacities. No morphological or molecular alterations in the endometrium that could interfere with the implantation of the fertilized egg have been demonstrated. There is no actual scientific evidence available supporting that the use of levonorgestrel for EOC is abortive.
\end{abstract}

Palabras clave: Postcoital contraception; Levonorgestrel; Endometrium; Ovulation; Spermatozoa (source: MeSH NLM).

\section{INTRODUCCIÓN}

El Ministerio de Salud de Perú (MINSA) establece que "... la salud reproductiva incluye la capacidad de las personas para tener una vida sexual segura y satisfactoria, para reproducirse $y$, la libertad para decidir cuándo y cómo y con qué frecuencia hacerlo ..." (1). En el año 2000, las mujeres peruanas en unión tenían en promedio
1,1 hijos más de lo deseado. El $31 \%$ de los nacimientos ocurridos en los últimos cinco años no fueron deseados y el $69,9 \%$ de las mujeres no usan métodos anticonceptivos (2). Cualquier intervención para superar la pobreza debiera considerar que muchas veces un embarazo no deseado a edades tempranas inicia el ciclo de transmisión intergeneracional de la pobreza ${ }^{(3)} \mathrm{y}$, por lo tanto, debe incluir la provisión de métodos anticonceptivos seguros y efectivos ${ }^{(4)}$.

Centro Nacional de Salud Pública, Instituto Nacional de Salud, Lima, Perú.

a Médico cirujano; ${ }^{b}$ Especialista en Enfermedades Infecciosas y Tropicales; ' Especialista en Epidemiología de Campo; ${ }^{d}$ Biólogo; e Magister en Biología Celular y Molecular; ${ }^{\mathrm{f}}$ Magister en Salud Pública; ${ }^{9}$ Doctor en Salud Publica; ${ }^{\mathrm{h}}$ Magister (c) en Epidemiología Clínica.

Recibido: 13-04-10 Aprobado: 02-06-10 
EI MINSA, en el marco de los lineamientos de los derechos de la salud sexual y reproductiva, pone a disposición de la población diversos métodos anticonceptivos. A partir del año 2001 esta entidad incorporó el uso de la Anticoncepción Oral de Emergencia (AOE) dentro de la Norma de Planificación Familiar (1), en concordancia con el objetivo de reducción de mortalidad materna del Plan Nacional Concertado de Salud ${ }^{(5)}$.

La anticoncepción oral de emergencia se refiere a todos los métodos hormonales que se usan como emergencia, luego de una relación sexual sin protección, para evitar un embarazo no deseado. La efectividad del método es de alrededor del $85 \%{ }^{(1)}$. Su indicación se limita a las siguientes situaciones: violación, violencia sexual familiar, relación sexual sin protección, si no usa regularmente un método anticonceptivo, se rompió o se deslizó el condón, se le olvidó de tomar más de dos días la píldora de uso regular, no se aplicó el inyectable en su fecha, se expulsó la $\mathrm{T}$ de cobre, usaron el coito interrumpido, usaron el método del ritmo y si tienen dudas sobre su efectividad (1). No obstante, en muchos países existe la creencia de que podría tener un efecto abortivo ${ }^{(6)}$

La Federación Internacional de Ginecología y Obstetricia (FIGO) ha definido a la reproducción natural humana, el embarazo y el aborto provocado. La reproducción natural es definida como un "proceso que comprende la producción de gametos masculinos y femeninos y su unión durante la fertilización". El embarazo es la parte del "proceso que comienza con la implantación del "conceptus" en el seno de una mujer, y que termina con el nacimiento de un bebé o con un aborto". El "aborto provocado es definido como la interrupción del embarazo mediante el empleo de medicamentos o intervención quirúrgica, tras la implantación, antes que el feto se haya convertido viable de forma independiente (definición de nacimiento de la OMS: pasadas 22 semanas o más después de la menstruación)" (4).

En el Perú, la AOE es un tema de actualidad y ha generado debates y posiciones sobre su uso. En el año 2003 mediante Resolución Suprema N. ${ }^{\circ}$ 003-2003-SA, el Ministerio de Salud nombra a una comisión de alto nivel encargada de analizar y emitir un informe científico, médico y jurídico sobre la AOE, que concluyó con la recomendación de su uso en todo el país al no poderse "asignar efecto abortifaciente" (7).

Sin embargo, persisten dudas por parte de algunas instituciones con respecto al mecanismo de acción del levonorgestrel (LNG) y su potencial efecto abortivo. En consecuencia, nuestro objetivo es revisar la evidencia científica disponible sobre los mecanismos de acción del LNG utilizado como anticonceptivo oral de emergencia.

\section{MÉTODOS}

Se realizó una revisión sistemática de la literatura científica que tuvo como pregunta de investigación: ¿Cuál es el efecto del levonorgestrel sobre endometrio, ovario y espermatozoides como anticonceptivo oral de emergencia?

La búsqueda se realizó en las bases de datos Medline y Cochrane Library incluyendo artículos que fueron indizados hasta el 1 de abril de 2010; se contempló los siguientes criterios de elegibilidad:

- Participantes: humanos y animales.

- Intervención: uso de levonorgestrel como anticonceptivo oral de emergencia (AOE).

- Resultados: efectos del LNG en el endometrio, ovario y espermatozoides.

- Diseño de estudios: estudios experimentales en animales, estudios observacionales, ensayos clínicos.

- Idiomas: ingles, español, francés y portugués.

- Publicaciones: artículos publicados en revistas científicas.

\section{ESTRATEGIA DE BÚSQUEDA}

Como estrategia de búsqueda primaria se realizó una pesquisa en Medline usando los siguientes términos $\mathrm{MeSH}$ :

(1) "Contraception, postcoital"[MeSH] OR "contraceptives, postcoital, synthetic"[MeSH] OR "contraceptives, postcoital'[MeSH] OR "contraceptives, postcoital, hormonal"[MeSH] OR "contraceptives, postcoital, synthetic"[Pharmacological Action] OR "contraceptives, postcoital, hormonal'[Pharmacological Action] OR "contraceptives, postcoital'[Pharmacological Action].

(2) Levonorgestrel.

(3) Se combinó (1) AND (2).

El término MeSH "contraception, postcoital" incluye: "postcoital contraception", "fertility control, postcoital", "control, postcoital fertility", "postcoital fertility control", "emergency contraception", "contraception, emergency"

Como estrategia de búsqueda secundaria se revisó las referencias contenidas en los artículos seleccionados y si se encontraba artículos relevantes que no estaban contenidos dentro de la búsqueda inicial, fueron incluidos en la lista de estudios para revisión. No se efectuó búsqueda de literatura gris. 


\section{SELECCIÓN DE ESTUDIOS}

Se elaboró un listado de los artículos hallados en las bases de datos investigadas y se eliminaron aquellos repetidos. Posteriormente, dos investigadores en forma independiente y ciega revisaron los títulos y los resúmenes para seleccionar aquellos que cumplían con los criterios de elegibilidad propuestos. En caso de divergencias se discutía con un tercer investigador para tomar una decisión. Las publicaciones seleccionadas fueron revisadas a texto completo, durante la cual se verificó nuevamente si cumplían con los criterios de selección.

\section{RECOLECCIÓN DE DATOS}

En una base de datos se registraron las siguientes variables de cada artículo: autor, revista, año de publicación, país, objetivo, tipo de estudio, población, dosis y vía de administración, métodos de medición, efectos en endometrio, efectos en ovulación, efectos en espermatozoides, conflictos de interés y fuente de financiamiento.

Finalmente, se analizó los efectos del uso de LNG como AOE sobre el endometrio, la ovulación y los espermatozoides, de modo independiente. Se describe los resultados y principales conclusiones de cada uno de los estudios incluidos. Los estudios seleccionados utilizaron diferentes diseños (experimentales, analíticos) y sujetos de estudio (humanos, animales) lo que es un factor que impide una acumulación de resultados para realizar un metaanálisis.

\section{RESULTADOS}

\section{ARTÍCULOS SELECCIONADOS PARA EL ANÁLISIS}

En Medline se encontró 480 referencias, cuyos títulos y resúmenes fueron revisados. Se excluyó artículos que tenían como objetivo evaluar la eficacia o seguridad del LNG como anticonceptivo oral de emergencia. También se excluyó los artículos que describían la farmacocinética del LNG y aquellos que evaluaban los efectos de una combinación de LNG con otra hormona. Se seleccionó sólo artículos originales; las revisiones fueron excluidas de este análisis. En la revisión de la bibliografía de estos artículos se encontró dos artículos originales adicionales. Finalmente fueron seleccionados 22 artículos (Figura 1).

De estos 22 artículos, publicados entre 1995 y el 2010, diez estuvieron diseñados para medir el efecto sobre la ovulación ${ }^{(8-17)}$, doce el efecto sobre el endometrio ${ }^{(9,12,13,18-}$ ${ }^{26)} y$ tres el efecto sobre los espermatozoides ${ }^{(27-29)}$. De estos estudios, nueve correspondían a ensayos clínicos

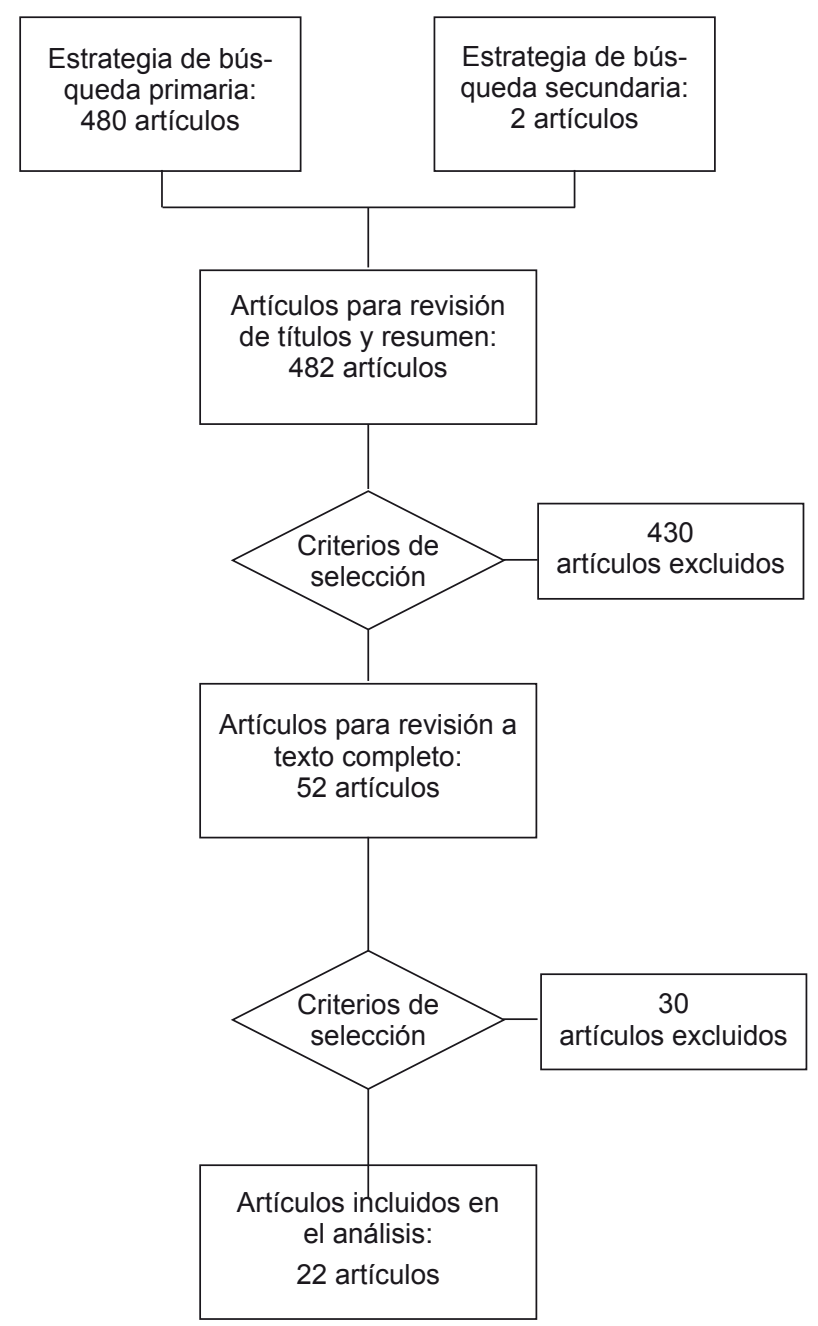

Figura 1. Proceso de selección de los artículos a ser incluidos en el análisis.

$(8,9,14,15,18,19,23,24,27)$, ocho a estudios cuasi-experimentales $(10-13,16,25,26,29)$, tres a estudios observacionales ${ }^{(17,22,28)}$ y dos a estudios in vitro ${ }^{(20,21)}$. Tres estudios fueron hechos en animales (los tres correspondientes a diseños cuasiexperimentales ${ }^{(13,16,25)}$ y el resto en humanos.

\section{EFECTOS SOBRE LA OVULACIÓN}

Fueron diez artículos los que evaluaron el efecto del LNG sobre la ovulación. Los estudios apoyan la hipótesis que la efectividad del LNG depende del momento de la administración con respecto a la ovulación. Cuando es administrado antes de la ovulación logra reducir la probabilidad de embarazos ${ }^{(14)}$. Su mecanismo de acción esta relacionado con la reducción de los niveles séricos de LH y FSH antes de la ovulación causando una ausencia de ovulación o su postergación (9-12,15,17). La efectividad en evitar la ruptura folicular también esta relacionada con el tamaño del folículo ${ }^{(8)}$. 
Los estudios realizados en animales también muestran que el LNG administrado tempranamente en el período preovulatorio es efectivo en suprimir o atrasar la ovulación $(13,16)$. La efectividad parece ser dependiente solo del momento de aplicación con respecto a la ovulación. Al igual que en humanos, se observa que si se administra muy cerca de la ovulación, la tasa de embarazo es igual que en el grupo control (16).

La Tabla 1 describe con detalle los resultados en la ovulación de los estudios realizados en humanos y animales con LNG.

\section{EFECTOS SOBRE EL ENDOMETRIO}

El efecto del LNG sobre la ovulación ha sido ampliamente documentado y los investigadores no tienen mayor discusión al respecto. Son los efectos sobre el endometrio los que han generado más discusión, principalmente por el riesgo que pueda perturbar la implantación del embrión. Para resolver este problema se han realizado estudios en humanos y animales.

Los estudios realizados para evaluar si hay variación en los receptores endometriales posterior a la medicación con LNG no han demostrado diferencias con respecto a los grupos control (12,18,19,21-24). Los principales receptores están relacionados con la progesterona. Otros receptores evaluados son los relacionados con los estrógenos, andrógenos, factor inhibidor de leucemia, interleukina $1 \beta$, factor de crecimiento endometrial vascular, ciclooxigenasa $1 \mathrm{y}$
2, y otros marcadores. A nivel estructural, Meng ${ }^{(23)}$ y Palomino ${ }^{(24)}$ hallaron leves cambios estructurales pero no hubo cambios en la expresión de los receptores endometriales. Ugocsai (26) reportó modificaciones más significativas pero las pacientes habían recibido dosis no usuales (cuatro a seis veces más elevadas que lo normal) de LNG.

Con respecto a la adhesión del blastocisto a las células endometriales, Lalitkumar ${ }^{(20)}$ realizóunestudioexperimental con células endometriales cultivadas y expuestas a LNG. Con respecto al grupo control, no observó diferencias en la adhesión de los blastocistos a las células. Dos estudios experimentales en ratas también evaluaron el efecto del LNG en la implantación de los blastocistos ${ }^{(13,25)}$. Para ello les administraron LNG, las aparearon y posterior a las fechas probables de implantación sacrificaron a los animales de experimentación. Los dos estudios no observaron diferencias en el número de implantaciones en el endometrio con relación al control. Sin embargo, Shirley ${ }^{(25)}$ describió que en el grupo que recibió una dosis más alta de LNG (5 mg) sí observó reducción significativa del número de implantaciones.

La Tabla 2 escribe los resultados en el endometrio de los estudios realizados en humanos y animales con levonorgestrel.

\section{EFECTOS SOBRE LOS ESPERMATOZOIDES}

Tres artículos refieren resultados explícitos sobre efectos en los espermatozoides. Un artículo reporta

Tabla 1. Artículos que evalúan los efectos del levonorgestrel en la ovulación.

\begin{tabular}{|c|c|c|c|c|}
\hline $\begin{array}{l}\text { Autor } \\
\text { (año) }\end{array}$ & $\begin{array}{l}\text { Tipo de } \\
\text { estudio }\end{array}$ & $\begin{array}{c}\text { Dosis de } \\
\text { levonorgestrel }\end{array}$ & Participantes & Resultados evaluados \\
\hline $\begin{array}{l}\text { Tirelli A, et al. } \\
(2008)^{(17)}\end{array}$ & $\begin{array}{l}\text { Descriptivo } \\
\text { prospectivo }\end{array}$ & $\begin{array}{l}0,75 \mathrm{mg} \text { en dos } \\
\text { dosis, cada } 12 \mathrm{~h}\end{array}$ & $\begin{array}{l}\text { Administración de LNG en fase folicular: } 26 \\
\text { mujeres, fase periovulatoria: } 14 \text {, fase luteal: } \\
29\end{array}$ & $\begin{array}{l}7 \text { de } 8 \text { mujeres que tomaron LNG en los días } \\
11 \text { a } 13 \text { del ciclo menstrual tuvieron un ciclo } \\
\text { anovulatorio sin elevación de LH y FSH }\end{array}$ \\
\hline $\begin{array}{l}\text { Novikova } N \text {, et } \\
\text { al. }(2006)^{(14)}\end{array}$ & $\begin{array}{l}\text { Descriptivo } \\
\text { prospectivo }\end{array}$ & $\begin{array}{l}1,5 \mathrm{mg} \text { en dosis } \\
\text { unica }\end{array}$ & $\begin{array}{l}51 \text { mujeres que tuvieron contacto sexual } \\
\text { no protegido entre los días }-5 \text { y } 0 \text { (día de la } \\
\text { ovulación) }\end{array}$ & $\begin{array}{l}17 \text { mujeres recibieron LNG después del día } \\
0 \text { y se observaron tres embarazos (dentro de } \\
\text { lo esperado según estimaciones). } 34 \text { mujeres } \\
\text { recibieron LNG antes o en el día } 0 \text { y no se } \\
\text { observaron embarazos (se esperaban cuatro } \\
\text { embarazos según estimaciones) }\end{array}$ \\
\hline $\begin{array}{l}\text { Croxatto HB, et } \\
\text { al. }(2004)^{(8)}\end{array}$ & $\begin{array}{l}\text { Ensayo } \\
\text { clínico }\end{array}$ & $\begin{array}{l}0,75 \mathrm{mg} \text { en dos } \\
\text { dosis, cada } 12 \\
\text { horas y } 1,5 \mathrm{mg} \\
\text { en dosis única }\end{array}$ & $\begin{array}{l}\text { En tres ciclos diferentes recibieron los dos } \\
\text { esquemas de LNG y uno de placebo. } 18 \\
\text { mujeres recibieron la medicación cuando el } \\
\text { folículo tenía un diámetro de } 12-14 \mathrm{~mm}, 22 \\
\text { con } 15-17 \mathrm{~mm} \text { y } 18 \text { con } \geq 18 \mathrm{~mm} \text {. }\end{array}$ & $\begin{array}{l}\text { La ausencia de ruptura folicular o disfunción } \\
\text { ovulatoria fue más frecuente }(p<0,05) \text { al recibir } \\
\text { LNG que al recibir placebo cuando el folículo } \\
\text { tenia un diámetro de } 12-14 \mathrm{~mm} \text { (dosis única) o } \\
\text { de } 15-17 \mathrm{~mm} \text { (dosis única o dos dosis). }\end{array}$ \\
\hline
\end{tabular}




\begin{tabular}{|c|c|c|c|c|}
\hline $\begin{array}{l}\text { Autor } \\
\text { (año) }\end{array}$ & $\begin{array}{l}\text { Tipo de } \\
\text { estudio }\end{array}$ & $\begin{array}{c}\text { Dosis de } \\
\text { levonorgestrel }\end{array}$ & Participantes & Resultados evaluados \\
\hline $\begin{array}{l}\text { Marions L, et } \\
\text { al. }(2004)^{(11)}\end{array}$ & $\begin{array}{l}\text { Estudio } \\
\text { cuasi expe- } \\
\text { rimental }\end{array}$ & $\begin{array}{l}0,75 \mathrm{mg} \text { en dos } \\
\text { dosis, cada } 12 \mathrm{~h}\end{array}$ & $\begin{array}{l}\text { Siete mujeres recibieron LNG y mifepristone } \\
\text { dos días antes de la ovulación en ciclos } \\
\text { diferentes con un ciclo libre entre los dos. }\end{array}$ & $\begin{array}{l}\text { Los niveles de LH fueron menores en los ciclos } \\
\text { que recibieron tratamiento que en los ciclos } \\
\text { control }(p<0,01) \text {. En ninguno de los ciclos con } \\
\text { tratamiento se observó ruptura del folículo. }\end{array}$ \\
\hline $\begin{array}{l}\text { Marions L, et } \\
\text { al. }(2002)^{(12)}\end{array}$ & $\begin{array}{l}\text { Estudio } \\
\text { cuasi expe- } \\
\text { rimental }\end{array}$ & $\begin{array}{l}0,75 \mathrm{mg} \text { en dos } \\
\text { dosis, cada } 12 \\
\text { horas }\end{array}$ & $\begin{array}{l}12 \text { mujeres recibieron cuatro tratamientos en } \\
\text { ciclos diferentes separados por un ciclo libre. } \\
\text { LNG fue administrado dos días antes de la } \\
\text { ovulación en un ciclo y dos días después de } \\
\text { la ovulación en otro ciclo. Mifepristone fue } \\
\text { administrado de forma similar. }\end{array}$ & $\begin{array}{l}\text { En los ciclos con LNG administrado antes } \\
\text { de la ovulación, los niveles séricos de LH no } \\
\text { mostraron diferencias con los demás días } \\
(p=0,77) ; \text { en los ciclos con LNG administrado } \\
\text { después de la ovulación, el patrón de LH sérico } \\
\text { fue similar a los ciclos control. }\end{array}$ \\
\hline $\begin{array}{l}\text { Durand M, et } \\
\text { al. }(2001)^{(9)}\end{array}$ & $\begin{array}{l}\text { Ensayo } \\
\text { clínico }\end{array}$ & $\begin{array}{l}0,75 \mathrm{mg} \text { en dos } \\
\text { dosis, cada } 12 \mathrm{~h}\end{array}$ & $\begin{array}{l}\text { Grupo A: } 15 \text { mujeres recibieron LNG el día } \\
10 \text { del ciclo menstrual. Grupo B: } 11 \text { mujeres } \\
\text { cuando se detecto LH en orina. Grupo C: } 11 \\
\text { mujeres a las } 48 \text { horas de la detección de } \\
\text { LH en orina. Grupo D: } 8 \text { mujeres en la fase } \\
\text { folicular tardía. El ciclo previo no tratado de } \\
\text { todas las mujeres fue el grupo control. }\end{array}$ & $\begin{array}{l}12 \text { mujeres del grupo A no ovularon. Las otras } \\
\text { tres mostraron alargamiento de la fase folicular } \\
\text { con respecto al control }(p<0,05) \text {, mientras que } \\
\text { los grupos } B, C \text { y } D \text { no mostraron diferencias. }\end{array}$ \\
\hline $\begin{array}{l}\text { Hapangama D, } \\
\text { et al. }(2001)^{(10)}\end{array}$ & $\begin{array}{l}\text { Estudio } \\
\text { cuasi expe- } \\
\text { rimental }\end{array}$ & $\begin{array}{l}0,75 \mathrm{mg} \text { en dos } \\
\text { dosis, cada } 12 \mathrm{~h}\end{array}$ & $\begin{array}{l}12 \text { mujeres recibieron LNG antes o en el día } \\
\text { que se detecto LH en la orina. Hubo ciclos } \\
\text { placebo. }\end{array}$ & $\begin{array}{l}\text { Una mujer no ovuló, en cuatro se postergó } \\
\text { significativamente el pico de LH; en ocho } \\
\text { las concentraciones de LH en la fase fueron } \\
\text { reducidas significativamente. }\end{array}$ \\
\hline \multicolumn{5}{|c|}{ Estudios en animales } \\
\hline $\begin{array}{l}\text { Ortiz ME, et al. } \\
(2004)^{(16)}\end{array}$ & $\begin{array}{l}\text { Estudio } \\
\text { cuasi expe- } \\
\text { rimental }\end{array}$ & $\begin{array}{l}0,75 \mathrm{mg} \text { oral o } \\
\text { subcutánea }\end{array}$ & $\begin{array}{l}\text { Efecto en la fertilidad: } 12 \text { hembras con dos } \\
\text { ciclos de tratamiento administrados muy } \\
\text { cerca de la ovulación y dos más con placebo. } \\
\text { Los tratamientos fueron administrados } \\
\text { después del apareamiento en la fase } \\
\text { periovulatoria. Efecto en la ovulación: seis } \\
\text { hembras con dos ciclos de tratamiento } \\
\text { administrados cuando el folículo era }<5 \mathrm{~mm} \\
\text { y dos ciclos cuando era } \geq 5 \mathrm{~mm} \text {; un ciclo } \\
\text { adicional se trato con placebo con el folículo } \\
<5 \mathrm{~mm}\end{array}$ & $\begin{array}{l}\text { Efecto en la fertilidad: la tasa de embarazo } \\
\text { fue idéntica en el grupo tratado y en el grupo } \\
\text { control }(54,2 \%) \text {. Efecto en la ovulación: en las } \\
\text { hembras tratadas con un folículo }<5 \mathrm{~mm} \text {, la } \\
\text { ovulación se suprimió ( } 4 / 12) \text { o se atrasó }(8 / 12) \text {; } \\
\text { en las hembras tratadas con un folículo } \geq 5 \mathrm{~mm} \\
\text { y las tratadas con placebo no hubo supresión ni } \\
\text { atraso de la ovulación. }\end{array}$ \\
\hline $\begin{array}{l}\text { Muller AL, et } \\
\text { al. }(2003)^{(13)}\end{array}$ & $\begin{array}{l}\text { Estudio } \\
\text { cuasi ex- } \\
\text { perimental }\end{array}$ & $\begin{array}{l}50 \mu \mathrm{g} \times \mathrm{kg} \\
\text { inyectable }\end{array}$ & Ratas hembras, dos a ocho por grupo & $\begin{array}{l}\text { Las hembras tratadas preovulatoriamente } \\
\text { (diestrus) tuvieron una proporción menor de } \\
\text { ovulación, } 25 \% \text {, que el grupo control, } 88 \% \\
\text { ( } p=0,02 \text { ). No hubo diferencias en la proporción } \\
\text { de hembras ovulando entre el grupo que recibió } \\
\text { tratamiento en la fase de crecimiento folicular } \\
\text { (proestrus) que el grupo control. }\end{array}$ \\
\hline
\end{tabular}

evidencia de la disminución del número de espermatozoides con el uso de levonorgestrel (28). Un estudio in vitro encontró que el LNG afecta directamente la velocidad curvilínea y lineal de los espermatozoides a dosis de 10 y de $100 \mathrm{ng}$. La velocidad promedio y lineal sólo fueron afectados por $100 \mathrm{ng} / \mathrm{mL}$ de LNG ${ }^{(29)}$.
También se ha reportado que no hay diferencias significativas en la cantidad de espermatozoides recuperados del útero en las 24 y 48 horas después del uso de LNG y no se evidencian cambios en la reacción acrosómica (capacidad del espermatozoide en penetrar al óvulo) ${ }^{(27)}$. 
Tabla 2. Artículos que evalúan los efectos del levonorgestrel (LNG) en el endometrio.

\begin{tabular}{|c|c|c|c|c|}
\hline $\begin{array}{l}\text { Autor } \\
\text { (año) }\end{array}$ & $\begin{array}{l}\text { Tipo de } \\
\text { estudio }\end{array}$ & $\begin{array}{c}\text { Dosis de } \\
\text { levonorgestrel }\end{array}$ & Participantes & Resultados evaluados \\
\hline \multicolumn{5}{|c|}{ Estudios en humanos } \\
\hline $\begin{array}{l}\text { Meng CX, et } \\
\text { al. }(2010)^{(23)}\end{array}$ & $\begin{array}{l}\text { Ensayo } \\
\text { clínico }\end{array}$ & $\begin{array}{l}0,75 \mathrm{mg} \text { oral por } \\
4 \text { veces cada } 24 \\
\text { horas vs } 1,5 \mathrm{mg} \text { vía } \\
\text { vaginal una vez }\end{array}$ & $\begin{array}{l}8 \text { mujeres en el grupo de adminis- } \\
\text { tración oral y } 7 \text { en el de administra- } \\
\text { ción vaginal; las mujeres tuvieron } \\
\text { un ciclo control y uno o dos ciclos } \\
\text { con tratamiento. }\end{array}$ & $\begin{array}{l}\text { Con la administración oral se observo una disminución de } \\
\text { la tinción de los receptores de progesterona (PR-A y PR-B) } \\
\text { en epitelio glandular en relación a los controles }(p=0,03) \\
\text { pero no en el estroma endometrial o epitelio luminal, sin } \\
\text { embargo no hubo cambios en la expresión de mRNA de } \\
\text { los PR. Se observó también un aumento la inmunoreacti- } \\
\text { vidad del factor inhibitorio de leucemia, confirmado por el } \\
\text { incremento de mRNA ( } p=0,03) \text {. No se observó cambios en } \\
\text { los otros receptores endometriales. Con la administración } \\
\text { vaginal no se observó ningún cambio en los receptores } \\
\text { endometriales. }\end{array}$ \\
\hline
\end{tabular}

$\begin{array}{ll}\text { Cultivos de células } \\ \text { Meng CX, et Estudio in } \\ \text { endometriales incu- } \\ \text { al. (2009) (21) vitro } & \begin{array}{l}\text { badas con proges- } \\ \text { terona, mifepristone } \\ \text { o LNG }\end{array}\end{array}$
o LNG

$\begin{array}{ll}\text { Meng CX, et } & \begin{array}{l}\text { Estudio } \\ \text { observa- } \quad 1,5 \text { mg vía oral. } \\ \text { al. (2009) }\end{array} \\ \text { cional }\end{array}$

Nueve mujeres que concibieron después de usar LNG post ovulatorio y decidieron abortar; nueve mujeres embarazadas no expuestas fueron el control.

Palomino

WA, et al.

$(2009)^{(24)}$

Ensayo

$1,5 \mathrm{mg}$ en una dosis

clínico

única vía oral y

vaginal

14 mujeres esterilizadas recibieron LNG por vía oral y 13 por vía vaginal.

Las células expuestas a levonorgestrel, con respecto a las células control, no mostraron cambios en la expresión de

los marcadores endometriales de receptividad estudiados: receptores de estrógeno, progesterona, andrógeno, factor inhibidor de leucemia, interleukina $1 \beta$, factor de crecimiento endometrial vascular, ciclooxigenasa 2 , factor de necrosis tumoral $\alpha$.

No hubieron diferencias significativas en la expresión de receptores de estrógenos (ERa, ER $\beta)$, progesterona $\left(\mathrm{PR}_{\mathrm{B}}\right.$, $\mathrm{PR}_{\mathrm{A}+\mathrm{B}}$ ), andrógenos y Ki67, entre el grupo tratado y el grupo control.

La evaluación histológica reveló pequeñas áreas de atrofia glandular y decidualizacion estromal intensa en sólo 3 de 12 biopsias en mujeres que tomaron LNG. A pesar de ello no se observaron cambios importantes en la reducción de la expresión de biomarcadores moleculares de receptividad del endometrio.

LNG no afecta la adhesión de blastocitos en el constructo Cultivos de células

Lalitkumar PG, et al. endometriales incu$(2007)^{(20)}$

Estudio in vitro terona, mifepristone o LNG

Células endometriales cultivadas a partir de las biopsias de endometrio de 12 mujeres. ndometrial in vitro, comparado con los controles. Mifepristona inhibe la adhesión del blastocisto en constructo endometrial in vitro comparado con los controles $(p<0,01)$.

Grupo 1: 8 mujeres recibieron LNG previo al pico de LH; grupo 2: 11

Durand $\mathrm{M}$, et Ensayo $0,75 \mathrm{mg}$ en dos doal. (2005) (19) clínico sis, cada 12 horas mujeres recibieron durante el pico de LH; grupo 3: 11 mujeres recibieron 48 horas después del pico de LH. El ciclo previo de todas las participantes fue el ciclo control.
En el grupo 1, los niveles séricos de glicodelina se elevaron más tempranamente y la expresión endometrial fue más débil en comparación con los otros grupos y el ciclo control.
8 mujeres recibieron levonorgestresl el dia $\mathrm{LH}+2$, previo a una cirugía de esterilización; ocho mujeres recibieron mifepristone; 8 mujeres fueron el grupo control.

12 mujeres recibieron cuatro tratamientos en ciclos diferentes sepa-

rados por un ciclo libre. LNG fue
Estudio

Marions L, et cuasi al. (2002) ${ }^{(12)}$ experimental
$0,75 \mathrm{mg}$ en dos dosis, cada 12 horas la ovulación en un ciclo y dos días después de la ovulación en otro ciclo. Mifepristone fue administrado de forma similar.

Estudio $\quad 0,75 \mathrm{mg}$ vía oral en 3 mujeres que consumieron LNG Ugocsai G, et cuasi al. (2002) (26) experimental varias dosis ( 4 a 6 en dosis altas de LNG y dos mujeveces más alto que res que no consumieron fueron el lo recomendado)
No se observó diferencias en la expresión de los receptores de estrógenos y de progesterona en las mujeres que recibieron LNG en comparación con el grupo control.

Con el uso de LNG no hubo diferencias en la expresión de receptores endometriales (COX-1, COX-2, receptores de progesterona, integrinaa 4 y $\beta 3$ ) con relación al grupo control.

Disminución notable de las células ciliadas endometriales durante la fase proliferativa y secretora en comparación con el grupo control. 


\begin{tabular}{|c|c|c|c|c|}
\hline $\begin{array}{l}\text { Autor } \\
\text { (año) }\end{array}$ & $\begin{array}{l}\text { Tipo de } \\
\text { estudio }\end{array}$ & $\begin{array}{l}\text { Dosis de } \\
\text { levonorgestrel }\end{array}$ & Participantes & Resultados evaluados \\
\hline $\begin{array}{l}\text { Durand M, et } \\
\text { al. }(2001)^{(9)}\end{array}$ & $\begin{array}{l}\text { Ensayo } \\
\text { clínico }\end{array}$ & $\begin{array}{l}0,75 \mathrm{mg} \text { en dos } \\
\text { dosis, cada } 12 \\
\text { horas }\end{array}$ & $\begin{array}{l}\text { Grupo A: } 15 \text { mujeres recibieron } \\
\text { LNG el día } 10 \text { del ciclo menstrual. } \\
\text { Grupo B: } 11 \text { mujeres cuando se } \\
\text { detectó LH en orina. Grupo C: } 11 \\
\text { mujeres a las } 48 \text { horas de la de- } \\
\text { tección de LH en orina. Grupo D: } 8 \\
\text { mujeres en la fase folicular tardía. } \\
\text { El ciclo previo no tratado de todas } \\
\text { las mujeres fue el grupo control. }\end{array}$ & $\begin{array}{l}\text { Nueve muestras fueron excluidas por ser insuficientes. No } \\
\text { hubo cambios significativos entre los tratados y las mues- } \\
\text { tras de control en cualquiera de las parámetros estudiados } \\
\text { ( } n^{\circ} \text { de glándulas por campo visual y por mm, edema estro- } \\
\text { mal, \% de tejido con edema estromal y arterias espirales } \\
\text { por número de campos visuales) }\end{array}$ \\
\hline \multicolumn{5}{|c|}{ Estudios en animales } \\
\hline $\begin{array}{l}\text { Muller AL, et } \\
\text { al. }(2003)^{(13)}\end{array}$ & $\begin{array}{l}\text { Estudio } \\
\text { cuasi } \\
\text { experi- } \\
\text { mental }\end{array}$ & $\begin{array}{l}50 \mu \mathrm{g} \times \mathrm{kg} \\
\text { inyectable }\end{array}$ & $\begin{array}{l}\text { Ratas hembras, dos a ocho por } \\
\text { grupo. }\end{array}$ & $\begin{array}{l}\text { No se observó diferencias significativas en el porcentaje de } \\
\text { huevos fertilizados ( } 75+/-14) \text { en comparación con el grupo } \\
\text { control ( } 97+/-3.3) \text {. Así mismo, no se encuentra diferencia } \\
\text { entre el número de embriones implantados en el grupo que } \\
\text { recibió LNG 15,3 +/- 0,6 versus el grupo control } 12,9+/-0,8 \text {. }\end{array}$ \\
\hline $\begin{array}{l}\text { Shirley B, et } \\
\text { al. (1995) (25) }\end{array}$ & $\begin{array}{l}\text { Estudio } \\
\text { cuasi } \\
\text { experi- } \\
\text { mental }\end{array}$ & $\begin{array}{l}1,5 \mathrm{mg} \text { y } 5 \mathrm{mg} \text { de } \\
\text { LNG subdérmicos }\end{array}$ & $\begin{array}{l}10 \text { ratas hembras en grupo de } 1,5 \\
\text { mg, } 15 \text { en grupo de } 5 \mathrm{mg} \text { y } 22 \text { en } \\
\text { grupo placebo. }\end{array}$ & $\begin{array}{l}\text { En el grupo de } 1,5 \mathrm{mg} \text {, no hubieron diferencias }(p>0,05) \\
\text { en el número de implantaciones en útero, numero de fetos } \\
\text { por hembra y resorciones por hembra embarazada con } \\
\text { relación al grupo control en el grupo de } 5 \mathrm{mg} \text {, no hubieron } \\
\text { implantaciones uterinas }(p<0,001) \text {. }\end{array}$ \\
\hline
\end{tabular}

\section{DISCUSIÓN}

Esta revisión de la literatura proporciona evidencias sobre los efectos del LNG en la ovulación, el endometrio y sobre los espermatozoides mediante estudios que han utilizado diferentes métodos de investigación. El efecto sobre la ovulación es bastante claro y ha sido descrito por varios autores. Administrado el LNG en días previos a la ovulación, puede inhibir o retrasar la ovulación mediante la supresión de los picos de LH y FSH. Este parece ser el mecanismo principal pues, si es administrado durante la ovulación o después, la tasa de embarazos es similar que en aquellas que no lo recibieron. En el endometrio, los receptores de progesterona y otros no muestran variaciones en su concentración después de la administración de LNG. Un estudio in vitro con células endometriales humanas y estudios en animales evidencian que la adhesión de los blastocitos al endometrio no varía con la administración del LNG. También es descrito un efecto sobre los espermatozoides, encontrándose un menor número de espermatozoides en el útero y disminución de la velocidad, pero no se observó alteraciones en la capacidad de penetrar al óvulo.

Hay varias revisiones publicadas con relación a este tema. Una revisión reciente ${ }^{(30)}$ describe que la efectividad observada en ensayos clínicos es mayor que la esperada si el único mecanismo de acción fuera la inhibición de la ovulación, lo cual sugiere que podría haber otros mecanismos implicados. Sin embargo, hay posibles explicaciones a esta diferencia, principalmente por el hecho que estos modelos de efectividad esperada se han elaborado sobre supuestos que necesitan ser validados. En cuanto a los efectos descritos en los diferentes estudios recopilados por la revisión mencionada, coincide en sus hallazgos con los nuestros. Otra revisión del tema ${ }^{(31)}$ no encuentra evidencia que el LNG afecte la receptividad endometrial y propone que estudios sobre la eficacia clínica del LNG descartan un efecto posfecundación, ya que mientras mayor es el intervalo entre el coito y la administración del LNG, menor es la eficacia. Otra revisión publicada sobre el LNG ${ }^{(32)}$ precisa que no hay efectos demostrables sobre las trompas de Falopio (no hay variación en la expresión de los receptores de estrógenos y progestágenos) ni sobre la etapa de la implantación (no hay alteración de los receptores endometriales y la eficacia del LNG es mucho menor comparada con métodos anti implantatorios como los dispositivos intrauterinos). Los autores postulan que en la etapa de la fertilización es posible que el LNG tenga algún efecto indirecto pues al ser administrada días antes del pico de LH produce concentraciones elevadas de glicodelina $\mathrm{A}$, la cual puede inhibir la unión del espermatozoide y el óvulo.

El uso del LNG como anticonceptivo oral de emergencia es cuestionado por la posibilidad que tenga un efecto abortivo. El aborto es definido como la pérdida del producto después de la implantación (4) y no hay evidencias que sugieran que el LNG pueda afectar la etapa de la implantación cuando es usado a las dosis convencionales. Los estudios en animales, los estudios in vitro, los estudios con biopsias endometriales y 
los estudios de eficacia clínica no han encontrado diferencias entre el uso del LNG y los controles con respecto a las diferentes variables evaluadas sobre el efecto en la implantación.

Los estudios revisados tienen ciertas limitaciones. En su mayoría, han tenido un pequeño número de participantes. Las variables evaluadas y las metodologías de medición son diferentes entre los estudios, lo cual hace difícil la acumulación de resultados. Sin embargo, estos diferentes enfoques y metodologías proporcionan una importante variedad de evidencias que se complementan entre sí.

Una limitación de la presente revisión es que no ha sido posible examinar todas las bases bibliográficas existentes e información publicada en idiomas distintos a los especificados. Sin embargo, como ya hemos descrito, las revisiones publicadas sobre el LNG coinciden con nuestros hallazgos y conclusiones. El presente artículo actualiza los hallazgos de las anteriores revisiones y se focaliza sobre los efectos del LNG con el fin de poder evaluar si tiene algún riesgo de ser abortivo, ya que esto puede condicionar la limitación de su uso en las políticas públicas de salud.

En conclusión, no existen evidencias sobre un posible efecto abortivo del levonorgestrel como anticonceptivo oral de emergencia. Estudios con un número más grande de participantes podrían ayudar a confirmar esta observación. Consideramos que el levonorgestrel puede formar parte de una política pública de salud reproductiva y sexual basada en evidencias que permita a las usuarias tomar una decisión informada adecuada.

\section{Fuente de Financiamiento}

Instituto Nacional de Salud

\section{Conflictos de Interés}

Los autores declaran no tener ningún conflicto de interés en la elaboración y publicación de este artículo.

\section{REFERENCIAS BIBLIOGRÁFICAS}

1. Perú, Ministerio de Salud. Norma Técnica de Planificación Familiar (N.T. No 032-MINSA/DGSP-V.01). Lima: MINSA; 2005.

2. Instituto Nacional de Estadística e Informática. Encuesta Demográfica y de Salud Familiar. Lima, INEI; 2005.

3. Alarcón G. ¿Cómo 'desconectar' la transmisión intergeneracional de la pobreza? El caso de las madres adolescentes en el Perú. Lima: INEI; 2006.

4. Federación Internacional de Ginecología y Obstetricia (FIGO), Comité Para El Estudio de los Aspectos Éticos de la Reproducción Humana. Recomendaciones sobre temas de ética en Obstetricia y Ginecología hechas por el Comité para el estudio de los aspectos éticos de la reproducción humana de la FIGO. Londres : FIGO; 2009.

5. Perú, Ministerio de Salud. Plan nacional concertado de salud. Lima: MINSA; 2007.

6. Organización Panamericana de la Salud (OPS), Programa Mujer, Salud y Desarrollo. Hoja informativa del Programa Mujer, Salud y Desarrollo sobre la Anticoncepción de Emergencia en las Américas. [Documento en internet] Washington DC, Organización Panamericana de la Salud. [fecha de acceso: 26 de febrero] Disponible en: http://www. paho.org/Spanish/AD/GE/emergencycontraceptionsp.pdf

7. Ministerio de Salud. Informe científico, médico y jurídico. Comisión de alto nivel -R.S.Nº07-2003-SA. Encargada de analizar y emitir informe final sobre la anticoncepción oral de emergencias (AOE). Lima, Ministerio de Salud; 2003.

8. Croxatto HB, Brache V, Pavez M, Cochon L, Forcelledo ML, Alvarez F, et al. Pituitary-ovarian function following the standard levonorgestrel emergency contraceptive dose or a single 0.75 -mg dose given on the days preceding ovulation. Contraception. 2004; 70(6): 442-50.

9. Durand M, del Carmen CM, Raymond EG, Durán-Sánchez O, De la Luz Cruz-Hinojosa M, Castell-Rodríguez A, et al. On the mechanisms of action of short-term levonorgestrel administration in emergency contraception. Contraception. 2001; 64(4): 227-34.

10. Hapangama D, Glasier AF, Baird DT. The effects of periovulatory administration of levonorgestrel on the menstrual cycle. Contraception. 2001; 63(3): 123-29.

11. Marions L, Cekan SZ, Bygdeman M, Gemzell-Danielsson K. Effect of emergency contraception with levonorgestrel or mifepristone on ovarian function. Contraception. 2004; 69(5): 373-77.

12. Marions L, Hultenby K, Lindell I, Sun X, Stabi B, Gemzell DK. Emergency contraception with mifepristone and levonorgestrel: mechanism of action. Obstet Gynecol. 2002; 100(1): 65-71.

13. Muller AL, Llados CM, Croxatto HB. Postcoital treatment with levonorgestrel does not disrupt postfertilization events in the rat. Contraception. 2003; 67(5): 415-19.

14. Novikova N, Weisberg E, Stanczyk FZ, Croxatto HB, Fraser IS. Effectiveness of levonorgestrel emergency contraception given before or after ovulation--a pilot study. Contraception. 2007; 75(2): 112-18.

15. Okewole IA, Arowojolu AO, Odusoga OL, Oloyede OA, Adeleye OA, Salu J, et al. Effect of single administration of levonorgestrel on the menstrual cycle. Contraception. 2007; 75(5): 372-77.

16. Ortiz ME, Ortiz RE, Fuentes MA, Parraguez VH, Croxatto HB. Post-coital administration of levonorgestrel does not interfere with post-fertilization events in the new-world monkey Cebus apella. Hum Reprod. 2004; 19(6): 1352-56.

17. Tirelli A, Cagnacci A, Volpe A. Levonorgestrel administration in emergency contraception: bleeding pattern and pituitary-ovarian function. Contraception. 2008; 77(5): 328-32.

18. Christow A, Sun X, Gemzell-Danielsson K. Effect of mifepristone and levonorgestrel on expression of steroid receptors in the human Fallopian tube. Mol Hum Reprod. 2002; 8(4): 333-40. 
19. Durand M, Seppala M, Cravioto MC, Koistinen H, Koistinen R, González-Macedo J, et al. Late follicular phase administration of levonorgestrel as an emergency contraceptive changes the secretory pattern of glycodelin in serum and endometrium during the luteal phase of the menstrual cycle. Contraception. 2005; 71(6): 451-57.

20. Lalitkumar PG, Lalitkumar S, Meng CX, Stavreus-Evers A, Hambiliki F, Bentin-Ley U, et al. Mifepristone, but not levonorgestrel, inhibits human blastocyst attachment to an in vitro endometrial three-dimensional cell culture model. Hum Reprod. 2007; 22(11): 3031-37.

21. Meng CX, Andersson KL, Bentin-Ley U, GemzellDanielsson K, Lalitkumar PG. Effect of levonorgestrel and mifepristone on endometrial receptivity markers in a threedimensional human endometrial cell culture model. Fertil Steril. 2009; 91(1): 256-64.

22. Meng CX, Cheng LN, Lalitkumar PG, Zhang L, Zhang HJ, Gemzell-Danielsson K. Expressions of steroid receptors and Ki67 in first-trimester decidua and chorionic villi exposed to levonorgestrel used for emergency contraception. Fertil Steril. 2009; 91(4 Suppl): 1420-23.

23. Meng CX, Marions L, Bystrom B, Gemzell-Danielsson K. Effects of oral and vaginal administration of levonorgestrel emergency contraception on markers of endometrial receptivity. Hum Reprod. 2010; 25(4): 874-83.

24. Palomino WA, Kohen P, Devoto L. A single midcycle dose of levonorgestrel similar to emergency contraceptive does not alter the expression of the L-selectin ligand or molecular markers of endometrial receptivity. Fertil Steril. 2009; Nov 10. [Epub ahead of print]

25. Shirley B, Bundren JC, McKinney S. Levonorgestrel as a postcoital contraceptive. Contraception. 1995; 52(5): 277-81.

26. Ugocsai G, Rozsa M, Ugocsai P. Scanning electron microscopic (SEM) changes of the endometrium in women taking high doses of levonorgestrel as emergency postcoital contraception. Contraception. 2002; 66(6): 433-37.
27. Do Nascimento JA, Seppala M, Perdigao A, EspejoArce X, Munuce MJ, Hautala $L$ et. al. In vivo assessment of the human sperm acrosome reaction and the expression of glycodelin-A in human endometrium after levonorgestrelemergency contraceptive pill administration. Hum Reprod. 2007; 22(8): 2190-95.

28. Espinos-Gomez JJ, Senosiain R, Mata A, Vanrell C, Bassas L, Calaf $\mathbf{J}$. What is the seminal exposition among women requiring emergency contraception? A prospective, observational comparative study. Eur J Obstet Gynecol Reprod Biol. 2007; 131(1): 57-60.

29. Yeung WS, Chiu PC, Wang $\mathrm{CH}$, Yao YQ, Ho PC. The effects of levonorgestrel on various sperm functions. Contraception. 2002; 66(6): 453-57.

30. Leung VW, Levine M, Soon JA. Mechanisms of action of hormonal emergency contraceptives. Pharmacotherapy. 2010; 30(2): 158-68.

31. Durand M, Larrea F, Schiavon R. Mecanismos de acción de la anticoncepción hormonal de emergencia: efectos del levonorgestrel anteriores y posteriores a la fecundación. Salud Publica Mex. 2009; 51(3): 255-61.

32. Saravi FD. Emergency contraception with levonorgestrel. Medicina (B Aires). 2007; 67(5): 481-90.

Correspondencia: Víctor J. Suárez.

Dirección: Cápac Yupanqui 1400, Lima 11, Perú.

Teléfono: (511) 617-6200 Anexo: 2143

Correo electrónico: vsuarez@ins.gob.pe ; vjsuarezm@yahoo.com

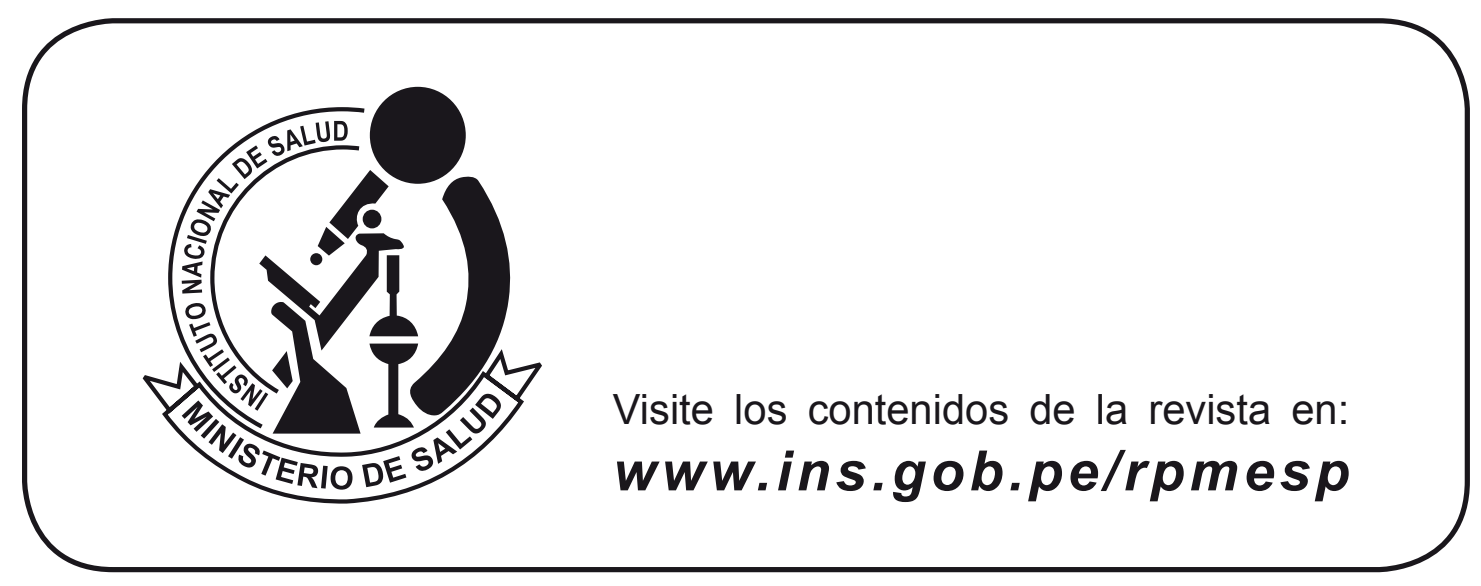

